\title{
PROPER TIME DERIVATIVES IN QUANTUM MECHANICS*
}

\author{
Juan P. APARICIO, Fabián H. GAIOLI, and Edgardo T. GARCIA ALVAREZ ${ }^{\dagger}$ \\ Departamento de Física, Facultad de Ciencias Exactas y Naturales, \\ Universidad de Buenos Aires, 1428 Buenos Aires, Argentina.
}

(September 4, 2018)

\begin{abstract}
Several quantum proper time derivatives are obtained from the Beck one in the usual framework of relativistic quantum mechanics (spin 1/2 case). The "scalar Hamiltonians" of these derivatives should be thought of as the conjugate variables of the proper time. Then, the Hamiltonians would play the role of mass operators, suggesting the formulation of an adequate extended indefinite mass framework. We propose and briefly develop the framework corresponding to the Feynman parametrization of the Dirac equation. In such a case we derive the other parametrizations known in the literature, linking the extension of the different proposals of quantum proper time derivatives again.
\end{abstract}

Pacs number: 03.65.Pm

*Published in Physical Review A 51, 96-103 (1995)

†Emails: gaiol@iafe.uba.ar, galvarez@dfuba.df.uba.ar 


\section{INTRODUCTION}

The proper time formulations of relativistic quantum mechanics (RQM) present some advantages with respect to the usual theory. The introduction of a "quantum proper time" 1 reestablishes in quantum theory the symmetry between space and time required by the special theory of relativity by elevating the coordinate $x^{0}$ to the rank of operator. This also permits us to recover a fundamental concept (a Lorentz scalar evolution parameter, the so-called "proper time") lost in the standard RQM. Thus putting in a parallel way both RQM and non-RQM, it allows us to use the well-known properties of this last theory. The main idea of a proper time formalism consists in taking states that evolve with a Schrödinger equation, whose "scalar Hamiltonian" plays the role of a mass operator. The standard theory can be recovered for definite mass states. Thus this framework provides a natural theoretical basis for a mass operator concept [1]. Furthermore, it allows solving the localization problem [2] by means of an extension of the Poincaré algebra, including a four "position" operator [3]. The Stückelberg [4] interpretation of antiparticles naturally arises in the formalism [5], which enables us to circumvent other related difficulties of the standard RQM [6], without appealing to quantum field theory 70 .

The idea of elevating the time coordinate $x^{0}$ to the rank of operator and of introducing the quantum analog of the classical proper time in RQM goes back to Dirac's earlier works 11]. These works have been forgotten, probably because Dirac himself did not insist on this point in his celebrated paper of 1928 [12]. However, from the pioneer works of Fock [13] and Stückelberg [4], many authors have made a considerable effort to develop a quantum theory with a proper time [14 17, 1, 1, 18 23, 3, 24, 37. In spite of this, the problem is still open because, among other questions, there exist different proposals and usually the authors present their versions omitting the relation to other approaches. The interpretation of the additional parameter also appears as a conceptual difficulty [5]. These topics are the core of this work.

In the majority of the contributions the quantum proper time is a $c$ number scalar parameter [38]. Many parametrizations or proper time derivatives have been used with some satisfactory results in each case. Some of them have used the formalism of the standard RQM, while others have extended it in several nonequivalent degrees.

The influence of Fock's 13 and Stückelberg's [4] papers is manifest; most authors have considered wave equations with second-order space-time derivatives

\footnotetext{
${ }^{1}$ This name is also used in the literature to refer to any invariant evolution parameter which under certain conditions can be related to the classical proper time.
}

(square mass operators) 15, 7, 1, 22, 26 31, 34, 35, 10, 37. In this line, we can also mention the classical paper of Schwinger [17], about the vacuum polarization and the electron anomalous magnetic moment. However, in this celebrated paper, the author makes use of the Stückelberg proper time formalism without stressing the physical interpretation of it. Later on, Roman et al. 26] have shown that such a parametrization can be seen as a representation of a five-dimensional Galilei group, introducing a universal length $l$ (in fact, this parametrization is essentially the Schwinger one if the evolution parameter is rescaled by choosing $l=2$ ).

On the other hand, Feynman [16] introduced a fifth parameter in the argument of the spinorial wave functions, considering the first-order Dirac equation 14, 16, 18, 19,24, 25, 33, 36. His approach to QED by means of the parametric Green's functions has many points in common with Schwinger's approach [39].

At this point it is natural to ask which is the relation among several results. In this work we investigate such a relation for the spin $1 / 2$ case and show why a one-particle indefinite mass theory is the appropriate framework. This is the case of Feynman's [16], Johnson's [3], and Schwinger's [17] proposals. With the help of physical restrictions we demonstrate that the first two parametrizations are equivalent and the evolution parameter introduced through them is reduced to the proper time. In contrast, the third parametrization, although it is a useful tool, does not lead to a classical theory in which the evolution parameter is reduced to the proper time. Thus the indefiniteness in the choice of a formalism whose evolution parameter was a suitable extension of the classical proper time is removed.

In Sec. II we show the relation among different proper time derivatives proposed in the literature in the framework of the standard definite mass theory. We propose, in Sec. III, an indefinite mass formalism and analyze the corresponding relations in such a context. For the sake of simplicity, we consider only, the minimal coupling case for electromagnetic interactions. The extension to more general interactions will be briefly discussed in Appendix B.

\section{PROPER TIME IN THE STANDARD DIRAC THEORY}

Although the introduction of a quantum proper time naturally leads to an indefinite mass theory in a direct way (see Sec. III), the standard framework of RQM (a definite mass theory) was frequently used by many authors. In this framework we can only work in a "formally covariant manner" since the temporal coordinate $t$ is a $c$ 
number. In such a case the inclusion of the proper time? is made through a quantum proper time derivative of the dynamical variables. This derivative is the counterpart of the ordinary time derivative of a dynamical variable $q$ in the Heisenberg picture, ${ }^{\beta}$ which reads

$$
\frac{d q}{d t}=i[H, q]+\frac{\partial q}{\partial t}
$$

with $H$ the Dirac Hamiltonian, i.e., for minimal coupling, $H=\vec{\alpha} \cdot \vec{\pi}+\beta m_{0}+e \phi$. If $H$ were replaced by a "scalar operator," we would obtain a quantum proper time derivative. For example, the Beck derivative 14 for a variable $q$, which does not explicitly depend on the proper time $s$, is defined by

$$
\left(\frac{d q}{d s}\right)_{B} \equiv-i[\mathcal{H}, q]
$$

where, for minimal coupling, $\mathcal{H}=\gamma^{\mu} \pi_{\mu}$ (with $\pi_{\mu}=p_{\mu}-$ $e A_{\mu}$ and $\left.p_{\mu}=i \partial_{\mu}\right)$. This is the natural choice. However, in most works the Fock 13,15 derivative is used:

$$
\left(\frac{d q}{d s}\right)_{F} \equiv-\frac{i}{2 m_{0}}\left[\mathcal{H}^{2}, q\right] .
$$

This is due to the following. While equations of motion with formal classical analogy are obtained by means of the Beck as well as the Fock derivatives, Beck's results do not seem to have such an analogy at first glance. This happens because the equations of motion actually describe "particle-antiparticle states" (classified by the sign of the mass) having covariant Zitterbewegung 41. Thus the nonpreference for the Beck derivative is due to the fact that the corresponding classical theory of spinning particles [42 is less known. We leave the discussion of this point for a separate paper, where we will study these equations in more detail [43].

In addition to the works of Fock and Beck, in the 1960s, Corben 20 proposed

$$
\left(\frac{d q}{d s}\right)_{C} \equiv \beta \frac{d q}{d t}
$$

as the quantum proper time derivative, inspired in the classical relation between $d s$ and $d t$, by identifying [(1$\left.\left.v^{2}\right)^{-1 / 2}\right]_{o p}$ with $\beta$. However, the Corben derivative does not satisfy Leibniz's rule 24]. He obtained some plausible results, but his hope to obtain a parallelism between

\footnotetext{
${ }^{2}$ The analogy with the classical counterparts derived from the equations of motion is the origin of the inaccurate name given to such a parameter.

${ }^{3}$ We use natural units $\hbar=c=1$ and the notations and conventions are those of Messiah 40.

${ }^{4}$ This fact cannot be interpreted in the context developed in this section. The adequate framework is an indefinite mass theory that we will introduce in Sec. III.
}

classical and quantum equations of motion of spinning particles was not completely successful.

In 1961, Fradkin and Good [21] considered

$$
\left(\frac{d q}{d s}\right)_{F G} \equiv\langle\beta\rangle \frac{d q}{d t}
$$

as a quantum proper time derivative, where $\langle\beta\rangle$ stands for the mean value of $\beta$. This proposal satisfies Leibniz's rule and has desirable properties in the classical limit. But it is strongly dependent on the quantum state 24.

Let us also remark that, up to now, the results obtained from the last derivatives are, in general, different (see Appendix A). In the following we will show a connection among them. So, even if some derivatives mentioned above were not good candidates in general, their results can be obtained from the Beck derivative.

Let us start by considering the Beck and the Corben derivatives. From definition (2) it follows that a dynamical variable $q$ satisfies

$$
\left(\frac{d q}{d s}\right)_{B}=\beta \frac{d q}{d t}+i[q, \beta]\left(i \frac{\partial}{\partial t}-H\right),
$$

where we have used that $\mathcal{H}$ can be rewritten as $\beta\left(i \partial_{t}-\right.$ $H)+m_{0}$ and Eq. (11).

If $[\beta, q]=0$, the Corben and the Beck derivatives are equivalent. In general $[\beta, q] \neq 0$, but these derivatives coincide when are applied to solutions $\phi(x)$ of the Dirac equation:

$$
\left(\frac{d q}{d s}\right)_{B} \phi(x)=\beta \frac{d q}{d t} \phi(x) .
$$

Let us note that, although the Corben derivative is a nonacceptable proposal, it works under some conditions. For example, the relation (7) explains why, in some cases, Corben had to restrict his equations to solutions of the Dirac equation.

With the help of (7) we can prove that, in the semiclassical limit, the Beck and the Fradkin-Good derivatives are equal in mean values. Moreover, the FradkinGood derivative was introduced precisely in this limit. In fact, considering the standard semiclassical coherent states, which factorize the mean value of the product of operators to first order in $\hbar$ [4], we have

$$
\left\langle\left(\frac{d q}{d s}\right)_{B}\right\rangle=\left\langle\left(\frac{d q}{d s}\right)_{C}\right\rangle=\langle\beta\rangle\left\langle\frac{d q}{d t}\right\rangle .
$$

Before establishing the connection between the Fock and the Beck derivatives, let us make a little digression about a new set of variables. They were introduced by Bunge and Kálnay 24 in an attempt to obtain a relativistic generalization of the Ehrenfest theorems. We will call them BK variables, which are defined by

\footnotetext{
${ }^{5}$ Notice that this identity is valid not only for minimal coupling, but also for the general case discussed in Appendix B.
} 


$$
Q \equiv q+\frac{i}{2 m_{0}}\left(\frac{d q}{d s}\right)_{B}
$$

where $q$ is a usual Dirac variable and $m_{0}$ is the mass of the particle. These variables were sometimes discussed in the literature. Thus, e.g.,

$$
X^{\mu}=x^{\mu}+\frac{i}{2 m_{0}} \gamma^{\mu}
$$

is a formally covariant position [45, 46, 18, 24, whose Beck derivative

$$
\left(\frac{d X^{\mu}}{d s}\right)_{B}=\frac{\gamma^{\mu}}{m_{0}}\left(m_{0}-\gamma^{\nu} \pi_{\nu}\right)+\frac{\pi^{\mu}}{m_{0}},
$$

is a BK variable and has curious properties 47 . The BK variable corresponding to the spin $\sigma^{\mu \nu}=(i / 2)\left[\gamma^{\mu}, \gamma^{\nu}\right]$ is the well-known Hilgevoord-Wouthuysen 48,49] spin tensor,

$$
\Sigma^{\mu \nu}=\sigma^{\mu \nu}-\frac{i}{m_{0}}\left(\gamma^{\mu} \pi^{\nu}-\gamma^{\nu} \pi^{\mu}\right),
$$

proposed originally to achieve a conserved spin in the free case.

On the other hand, if we consider the polarization operator defined by Michel and Wightman [50, $t^{\mu}=i \gamma^{5} \gamma^{\mu}$, the BK variable associated with it is

$$
T^{\mu}=i \gamma^{5}\left(\gamma^{\mu}-\frac{\pi^{\mu}}{m_{0}}\right) .
$$

This operator was introduced by Fradkin and Good 21] and it is such that, applied to solutions of the Dirac equation, it coincides with the polarization operators considered by Bargmann and Wigner (BW) [51] $\left(T_{B W}^{\mu}=\right.$ $\left.\frac{1}{2} \epsilon^{\mu \nu \rho \lambda} \sigma_{\nu \rho} \frac{\pi_{\lambda}}{m_{0}}\right)$ and by Kolsrud [49] $\left(T_{K}^{\mu}=\gamma_{5} \sigma^{\mu \nu} \frac{\pi_{\nu}}{m_{0}}\right)$.

Now, let us find the relation between the Fock and the Beck derivatives. Observe that

$$
\left(\frac{d X^{\mu}}{d s}\right)_{B} \phi(x)=\left(\frac{d x^{\mu}}{d s}\right)_{F} \phi(x)=\frac{\pi^{\mu}}{m_{0}} \phi(x) .
$$

where $\phi(x)$ is a solution of the Dirac equation. The last equality, which comes from Eq. (10), shows that the BK velocity has classical analogy [24, as it happens in the Fock case. Thus, from this equation it is tempting to find a general relation. In fact, a straightforward calculation of the Beck derivative of a BK variable leads to

\footnotetext{
${ }^{6}$ The BW and the Kolsrud operators are equal; it is easily checked by the identity $\frac{1}{2} \epsilon^{\mu \nu \rho \lambda} \sigma_{\rho \lambda}=\gamma_{5} \sigma^{\mu \nu}$. Using that $\gamma^{\mu} \gamma^{\nu}=g^{\mu \nu}-i \sigma^{\mu \nu}$, the Kolsrud operator can be rewritten in the form $T_{K}^{\mu}=i \gamma_{5}\left(\gamma^{\mu} \gamma^{\nu} \frac{\pi_{\nu}}{m_{0}}-\frac{\pi^{\mu}}{m_{0}}\right)$. When applied to solutions of the Dirac equation, the Kolsrud operator coincides with the operator of Eq. (11). We have used the conventions $\epsilon^{0123}=1$ and $\gamma^{5}=\gamma^{0} \gamma^{1} \gamma^{2} \gamma^{3}$.
}

$$
\left(\frac{d Q}{d s}\right)_{B}=-\frac{i}{m_{0}}[\mathcal{H}, q]\left(m_{0}-\mathcal{H}\right)-\frac{i}{2 m_{0}}\left[\mathcal{H}^{2}, q\right]
$$

Then, if $\phi(x)$ is a solution of the Dirac equation, we obtain the general result

$$
\left(\frac{d Q}{d s}\right)_{B} \phi(x)=\left(\frac{d q}{d s}\right)_{F} \phi(x) .
$$

The Beck derivative of BK variables coincides with the Fock derivative of ordinary variables applied to solutions of the Dirac equation. In the next section we prove that, in the framework of an indefinite mass theory, this relation is even closer. Summing up, we have seen in this section that from the Beck derivative, which is the natural extension of a proper time derivative, the results derived from all derivatives proposed in the literature can be obtained. Moreover, we will see in the next section that this derivative corresponds, in an adequate framework, to the Heisenberg picture of the Feynman parametrization of the Dirac equation.

\section{PROPER TIME IN INDEFINITE MASS THEORIES}

As we have already anticipated in the Introduction, a quantum proper time derivative can be introduced by first principles only in an indefinite mass theory. These theories have in common a Schrödinger equation in which the evolution parameter is the proper time and the role of the Hamiltonian is played by a scalar (mass) operator. A general solution of this equation has mass dispersion; thus results the name of such theories. It is important to remark that most works have considered spinless systems and used parametrizations with Hamiltonians quadratic in the momenta.

We shall revise the most well-known approaches for the spin 1/2 case. For example, in Schwinger's approach [17], the integral representation of the Green's function of the Dirac equation suggested the formal introduction of an evolution operator in a proper time $\lambda$ defined by $\mathcal{U}(\lambda)=e^{i \mathcal{H}^{2} \lambda}$. Thus the Heisenberg equation of motion for a variable $q$ not explicitly $\lambda$ dependent is

$$
\frac{d q}{d \lambda}=-i\left[\mathcal{H}^{2}, q\right]
$$

which we will call the Schwinger derivative.7 We can see that, in the classical limit, the evolution parameter $\lambda$ is unrelated to the proper time $s$. In fact, only after

\footnotetext{
${ }^{7}$ All definite mass derivatives were distinguished with different names, but identified with the same proper time parameter $s$. However, in indefinite mass theories, different letters are used for the evolution parameters when they are not directly related to the proper time.
} 
choosing the initial conditions on a given mass shell, 8 we have $\lambda=s /\left(2 m_{0}\right)$.

In recent works some authors have returned to this line [31,34, 35, 37] trying to provide a theoretical framework for this parametrization (for the spin 0 case). Observe that, in order to have a more direct identification of the evolution parameter with the classical proper time, the Schwinger Hamiltonian must be rescaled with a factor $1 /\left(2 m_{0}\right)$, as in the Fock derivative (Sec. II). However, as we shall see later, it is not easy to achieve such an identification without violating the indefinite mass character of the theory. The approaches known as relativistic dynamics (RD) [27,28] and four space formulation (FSF) 29,30 are attempts to give a foundation to a formalism of this kind. However, similar objections arise.

The proper time derivative of $\mathrm{RD}$ and FSF has a Hamiltonian which depends on an intrinsic mass parameter $M$,

$$
\frac{d q}{d \tau}=-\frac{i}{2 M}\left[\mathcal{H}^{2}, q\right],
$$

not related a priori with the mass $m_{0}$ of the particle, ? as it is desirable in an indefinite mass formalism. T0 However, in order that the evolution parameter $\tau$ becomes the proper time in the classical limit we must identify (on shell) $M$ with the mass $m_{0}$ a posteriori. The last identification seems to be an unattractive feature for an indefinite mass theory, since the Hamiltonian includes the information about some given initial conditions.

Finally, let us comment on Johnson's interesting approach [3,25]. In his first work [3], he considers all spins in the free case using a unique Hamiltonian $\mathcal{H}_{J} \equiv \sqrt{p^{\mu} p_{\mu}}$, which governs the evolution in a time $s .{ }^{11}$ The physical states belong to the subspace spanned by eigenfuctions of $\mathcal{H}_{J}$ with positive eigenvalues. In general, we define the extension of the Johnson Hamiltonian as

$$
\mathcal{H}_{J} \equiv \sqrt{\mathcal{H}^{2}} \equiv m_{o p},
$$

where, e.g., $\mathcal{H}^{2}=\pi^{\mu} \pi_{\mu}-(e / 2) \sigma^{\mu \nu} F_{\mu \nu}$ for minimal coupling. The Heisenberg evolution of a dynamical variable $q$, which does not explicitly depend on $s$, will be called the Johnson derivative

\footnotetext{
${ }^{8}$ Classically $\pi^{\mu} \pi_{\mu}$ is a constant of motion, which can be fixed to a particular value $m_{0}^{2}$.

${ }^{9}$ Strictly speaking, Eq. 16 is used by RD and FSF in the free case $\left(\mathcal{H}^{2}=p^{\mu} p_{\mu}\right)$. For the interaction case they use a slightly different formalism; see Ref. 28.

${ }^{10}$ Equation (16) is also the case of the Roman parametrization [26] identifying $M$ with the inverse of the universal length $l$.

${ }^{11}$ As we shall see in Sec. III.A, the evolution parameter of the corresponding classical theory (independently proposed by Moses for spin 0 23]) is the proper time.
}

$$
\left(\frac{d q}{d s}\right)_{J} \equiv-i\left[\sqrt{\mathcal{H}^{2}}, q\right] .
$$

We will not consider in more detail either the secondorder [Eqs. (15) and (16)] or square root [Eq. (18)] parametrizations. We will see how to relate these alternatives to a first-order parametrization whose dynamics is governed by the scalar Hamiltonian $\mathcal{H}$, originally proposed by Feynman [16].

\section{A. A proper time formulation of RQM based on a first-order parametrization}

The way to introduce the concept of proper time in the spin 1/2 RQM would be achieved within a framework which should satisfy at least these requirements: (a) Dirac's theory must be somehow included; (b) the equations of motion must be analogous to a classical theory in which the evolution parameter is the proper time; (c) the framework must not be restricted to the mass shell. Condition (a) is Bohr's correspondence principle in a broad sense, while (b) is the same principle applied to the relation between classical and quantum mechanics. It will allow us to identify the evolution parameter with the classical proper time. Condition (c) is what we have called an "indefinite mass theory," which is a consequence of extending the Poincaré algebra [23, 3, 26 in order to include a Dirac four-vector position operator $x^{\mu}$, the canonical conjugate of $p_{\mu}$,

$$
\left[x^{\mu}, p^{\nu}\right]=-i \hbar g^{\mu \nu} .
$$

In fact, just at the classical level, we can see that the mass constraint $\left(p^{\mu} p_{\mu}=m^{2}\right)$ is incompatible with the Poisson bracket corresponding to Eq. (19).

Taking condition (c) into account, which allows realizing the algebra (19), let us consider a wave function $\Psi(x, s)$ belonging to a linear space of spinorial functions defined in space-time. The wave function represents the state of the system at a given value of the parameter $s$ and its evolution is given by

$$
-i \frac{d}{d s} \Psi(x, s)=\mathcal{H} \Psi(x, s) .
$$

The scalar Hamiltonian $\mathcal{H}$ plays the role of the standard Hamiltonian in non-RQM. From (20), the evolution operator is

$$
U(s)=e^{i \mathcal{H} s} .
$$

Going to the Heisenberg picture, in which the evolution of the operators is governed by

$$
\frac{d q}{d s}=-i[\mathcal{H}, q],
$$

we reobtain the Beck equation of motion (2).

The eigenfunctions of $\mathcal{H}$ are definite mass states $\phi_{m}$ that satisfy the eigenvalue equation $\mathcal{H} \phi_{m}=m \phi_{m}$. They 
have oscillatory behavior in $s$ and are solutions of a generalized Dirac equation [condition (a)]. In addition, in the space of these spinorial wave functions, we define an indefinite Hermitian form [52 54]

$$
\langle\Phi \mid \Psi\rangle \equiv \int \bar{\Phi} \Psi d^{4} x
$$

where $\bar{\Phi}=\Phi^{\dagger} \gamma^{0}$ is the usual Dirac adjoint.

Observe that the spin variables $\gamma^{\mu}$, the spin tensor $\sigma^{\mu \nu}$, and the orbital variables $p_{\mu}$ and $x^{\mu}$, as well as the Hamiltonian, become Hermitian in the "scalar product" 23.). Hence the evolution operator (21) is unitary [5]. From this, the "norm" of the states is a constant of motion, i.e.,

$$
\frac{d}{d s}\langle\Psi \mid \Psi\rangle=0
$$

Thus the subspaces corresponding to the states with positive, negative, or null norms are invariant under the proper time evolution. Moreover, the standard interactions, e.g., the ones considered in this work, are $s$ independent and then they cannot produce transitions among states belonging to different subspaces.

Analogously to what happened in Sec. II, the equations of motion corresponding to different parametrizations are, in general, different too (see Appendix A). To establish a connection among them we will consider the restriction of the formalism on the positive mass subspace, i.e., the subspace invariant under the action of the projector defined by

$$
\Lambda \equiv \frac{1}{2}\left(1+\frac{\mathcal{H}}{m_{o p}}\right) .
$$

We will see that with this restriction the Feynman parametrization is equivalent to the Johnson one. First, let us note that in this subspace the Hamiltonians $\mathcal{H}$ and $\mathcal{H}_{J}$ coincide,

$$
\Lambda \mathcal{H} \Lambda=\Lambda \mathcal{H}_{J} \Lambda
$$

Observe also that

$$
[\mathcal{H}, \Lambda]=0=\left[\mathcal{H}_{J}, \Lambda\right], \quad \frac{d \Lambda}{d s}=0=\left(\frac{d \Lambda}{d s}\right)_{J},
$$

and therefore the action of the projector is invariant under the proper time evolution. From the definitions (22) and (18), for any variable $q$ we have

\footnotetext{
${ }^{12}$ It is a straightforward extension of the well known positive energy projector in the standard case. See, e.g., Ref. 40]. The projector 25 was also used by Johnson and Chang (see the second paper in Ref. 25]) and by Enatsu and Kawaguchi (see the second paper in Ref. (1).
}

$$
\Lambda \frac{d q}{d s} \Lambda=-i \Lambda\left[\sqrt{\mathcal{H}^{2}}, q\right] \Lambda=\Lambda\left(\frac{d q}{d s}\right)_{J} \Lambda
$$

Thus, in the subspace of positive mass, the Beck and the Johnson derivatives are equivalent.

Now, let us take the classical limit in order to visualize a closer connection among the different parametrizations discussed at the beginning of this section. In this way, we will also be able to identify the parameter $s$ with the proper time.

Let us first recall that if $A$ and $B$ are operators, and $f(B)$ is an operator function, it follows that

$$
[A, f(B)]=\frac{1}{2} \frac{d f}{d B}[A, B]+[A, B] \frac{1}{2} \frac{d f}{d B}+O\left(\hbar^{2}\right) .
$$

Therefore, to first order in $\hbar$, we have

$$
\Lambda \frac{d q}{d s} \Lambda=\Lambda \frac{1}{2}\left(\frac{1}{2 m_{o p}}\left[-i \mathcal{H}^{2}, q\right]+\left[-i \mathcal{H}^{2}, q\right] \frac{1}{2 m_{o p}}\right) \Lambda .
$$

Then, if we take mean values with a positive mass quasiclassical state $\mathrm{H}^{\mathrm{T}}$ and consider that these states factorize the operator product up to the first order in $\hbar,{ }^{14}$ we obtain

$$
\left\langle\frac{d q}{d s}\right\rangle=\left\langle\frac{1}{2 m_{o p}}\right\rangle\left\langle\frac{d q}{d \lambda}\right\rangle=\left\langle\frac{M}{m_{o p}}\right\rangle\left\langle\frac{d q}{d \tau}\right\rangle,
$$

where, in this limit, $m_{o p}=\sqrt{\pi^{\mu} \pi_{\mu}}$. This equation relates the Feynman parametrization with the Schwinger, RD, and FSF ones, in the classical limit. Observe that RD and FSF can only recover the results of the Feynman parametrization after choosing the initial conditions on the mass shell $\left\langle m_{o p}\right\rangle=M$.

From the first equality of Eq. (29), we obtain

$$
\frac{d}{d s}\left\langle x^{\mu}\right\rangle \frac{d}{d s}\left\langle x_{\mu}\right\rangle=\frac{\left\langle\pi^{\mu}\right\rangle\left\langle\pi_{\mu}\right\rangle}{\left\langle m_{o p}\right\rangle^{2}}=1,
$$

since $\left\langle m_{o p}\right\rangle^{2}=\left\langle\pi^{\mu}\right\rangle\left\langle\pi_{\mu}\right\rangle$ because of the factorization property. Thus $s$ is reduced to the proper time of indefinite mass states that follow the world line $\left\langle x^{\mu}\right\rangle(s)$.

We have seen that the formalism briefly sketched in this section permits us to obtain the desired results included in all the parametrizations, without appealing to ad hoc assumptions. Moreover, the equations of motion

\footnotetext{
${ }^{13}$ This means that $\Lambda|\rangle=[1+O(\hbar)]|\rangle$.

${ }^{14}$ The quasiclassical states considered in this case are different from those considered in the standard Dirac theory. In Dirac's theory, since time $t$ is a $c$ number, these states are essentially the same coherent states of non-RQM. In indefinite mass theories the role played by $t$ is very different. Then, the quasiclassical states must be generalized. A straightforward generalization is shown in Ref. [5].
} 
of the main dynamical variables derived from Eq. (29) read (in the free case)

$$
\begin{gathered}
\frac{d}{d s}\left\langle x^{\mu}\right\rangle=\frac{\left\langle p^{\mu}\right\rangle}{\left\langle m_{o p}\right\rangle}, \\
\frac{d}{d s}\left\langle p^{\mu}\right\rangle=0, \\
\frac{d}{d s}\left\langle\sigma^{\mu \nu}\right\rangle=0 .
\end{gathered}
$$

These equations on shell have a classical analogy; the four-velocity is proportional to the four-momentum, which results in a conserved quantity, as is the case of the spin tensor. This fulfills the outline of our proposal, since from (30) and (31) we satisfy condition (b).

In order to complete the picture of Sec. II let us see how to generalize the relation (12) from an extension of the BK variables. The BK variables used in the definite mass theory have no meaning in the indefinite mass case since they depend on a particular mass value. One way of bypassing such a difficulty consists in making the heuristic substitution $m_{0} \rightarrow m_{o p}$. So, we define a new BK variable as

$$
Q \equiv q+\frac{i}{2}\left(-i\left[\frac{\mathcal{H}}{m_{o p}}, q\right]\right)
$$

For example, the position operator reads

$$
X^{\mu}=x^{\mu}+\frac{i}{2 m_{o p}} \gamma^{\mu}-\frac{i p^{\mu}}{2 m_{o p}^{3}} \mathcal{H}
$$

in the free case. Here an extra term appears because, in general, the variable $q$ does not commute with $m_{o p}$ in (32). 15

Using Eq. (26) it is immediate that, for a dynamical variable $q$, we have

$$
\Lambda q \Lambda=\Lambda Q \Lambda
$$

and trivially, with (27) and (28),

$$
\Lambda \frac{d Q}{d s} \Lambda=\Lambda\left(\frac{d q}{d s}\right)_{J} \Lambda
$$

Then, the Johnson derivative ${ }^{16}$ of the Dirac variables coincides with the Beck derivative of the new BK variables, which constitutes the generalization of (14).

\footnotetext{
${ }^{15}$ The unification of the different proposals for the polarization operators shown in Sec. II can be easily transposed to this case.

${ }^{16}$ Notice that the Fock derivative becomes the Johnson one by means of the same heuristic substitution $m_{0} \longrightarrow m_{\text {op }}$.
}

\section{CONCLUDING REMARKS}

We have seen in this paper that there are different proposals to introduce a quantum proper time derivative in RQM, but they are not completely satisfactory. Recalling the conditions imposed to a proper time formulation of RQM in Sec. III.A, we can see that some proposals, e.g., RD and FSF, satisfy condition (a), but fail to give an adequate framework simultaneously compatible with conditions (b) and (c). A natural extended framework, which resembles the usual non-RQM, that satisfies conditions (a) and (c) was given in this paper by means of the Feynman parametrization (used by him as a formal tool for obtaining the famous results of QED in a heuristic way [16]). It has possibly been forgotten because apparently it does not verify condition (b). However, we have demonstrated in this work that the Feynman parametrization is compatible with condition (b). It also permits us to derive the other proposals known in the literature as particular cases, establishing a connection among them under adequate restrictions. Thus we have shown a unified version of different proposals of proper time derivative, both in the standard as well as in the extended (indefinite mass) framework of RQM.

Summing up, a unique parametrization of the spin $1 / 2$ "particle" (the natural one), which includes the standard formalism of RQM as a particular case, is enough in order to have an overall view of the different previous proposals.

\section{ACKNOWLEDGMENTS}

We are grateful to A.J. Kálnay for interesting and useful discussions. We would like to thank C.S. Bernardou for reading the manuscript. We are supported by the Universidad de Buenos Aires. 


\section{APPENDIX A:}

In Sec. II we stated that the results obtained through the several proposals of proper time derivatives are, in general, different. Let us see, as an example, only the velocities derived from them. These read

$$
\begin{gathered}
\left(\frac{d x^{\mu}}{d s}\right)_{B}=\gamma^{\mu}, \\
\left(\frac{d x^{\mu}}{d s}\right)_{F}=\frac{\pi^{\mu}}{m}, \\
\left(\frac{d x^{\mu}}{d s}\right)_{C}=\gamma^{\mu}, \\
\left(\frac{d x^{\mu}}{d s}\right)_{F G}=\langle\beta\rangle \beta \gamma^{\mu},
\end{gathered}
$$

which confirms the above statement.

On the other hand, in the indefinite mass case (Sec. III) we have, for the derivatives of the position operator,

$$
\begin{gathered}
\frac{d x^{\mu}}{d \lambda}=2 \pi^{\mu}, \\
\frac{d x^{\mu}}{d \tau}=\frac{\pi^{\mu}}{M}, \\
\left(\frac{d x^{\mu}}{d s}\right)_{J}=\frac{1}{2}\left(\pi^{\mu} \frac{1}{m_{o p}}+\frac{1}{m_{o p}} \pi^{\mu}\right)+O\left(\hbar^{2}\right), \\
\frac{d x^{\mu}}{d s}=\gamma^{\mu} .
\end{gathered}
$$

The corresponding statement is verified again.

\section{APPENDIX B:}

Let us comment on some extensions of the results of previous sections for the case of interactions more general than minimal coupling. Even if the great majority of the works about proper time have considered only the free case, and sometimes the minimal coupling case, the interest for nonminimal couplings and for nonelectromagnetic interactions has been revived recently in connection with the quantum equations of motion of spinning particles [55, 36, 43,56 .

Let us consider the scalar Hamiltonian

$$
\mathcal{H}_{R}=\gamma^{\mu} p_{\mu}-R
$$

where $R$ is an arbitrary operator which preserves the Lorentz and gauge invariance of the theory. If the different derivatives are adequately redefined, all relations presented in this work remain valid. It is easy to check this fact, since the results have been obtained without making use of the explicit form of $R$.

An example of a possible extension of the scalar Hamiltonian is

$$
\mathcal{H}_{R}=\sum_{n=0}^{\infty}\left[\epsilon_{n} \gamma^{\mu} \square^{n} A_{\mu}+\frac{i}{2} \mu_{n} \gamma^{\mu} \gamma^{\nu} \square^{n}\left(\partial_{\mu} A_{\nu}-\partial_{\nu} A_{\mu}\right)\right]
$$

which was originally proposed by Foldy [57]. For suitable choices of the coupling constants $\epsilon_{n}$ and $\mu_{n}$, e.g., the minimal $\left(\epsilon_{0}=e ; \epsilon_{n}=0, n \neq 0 ; \mu_{n}=0 \forall n\right)$ or the Pauli $\left(\epsilon_{n}=0 \forall n ; \mu_{0}=\chi \frac{e}{2 m_{0}} ; \mu_{n}=0, n \neq 0\right)$ couplings are obtained. The Dirac equation that results, retaining the three first terms, has been used frequently in the past in order to describe nucleons. Furthermore, we have proved recently that this equation provides a model to obtain the relativistic part of the radiative corrections of the energy levels of the electron in an external electromagnetic field 58 .

Alternatively, if we choose

$$
R=g \gamma^{\mu} A_{\mu}^{a} L_{a}
$$

where $g$ is a coupling constant, $A_{\mu}^{a}$ are Yang-Mills fields, and $L_{a}$ the corresponding generators of the gauge group, $\left[L_{a}, L_{b}\right]=i C_{a b}^{c} L_{c}$, we can reproduce the equations of motion of a "colored" spinning particle [55], as we will show in another paper [56].

Another possibility is 59

$$
R=e \gamma^{\mu} A_{\mu}+\mu_{0}^{(m)} \frac{e}{2 m_{0}} \sigma^{\mu \nu} F_{\mu \nu}+\mu_{0}^{(e)} \frac{e}{2 m_{0}} \gamma_{5} \sigma^{\mu \nu} F_{\mu \nu},
$$

which takes into account particles with anomalous moments [60] (including a possible time reversal violation). The last term can be thought of as a Pauli coupling containing the dual electromagnetic tensor (see the first identity of footnote 6 ), i.e., the case of a monopole Pauli coupling.

[1] H. Enatsu, Suppl. Nuovo Cimento 3, 526 (1956); Prog. Theor. Phys. 30, 236 (1963); H. Enatsu and S. Kawaguchi, Nuovo Cimento A 27, 458 (1975); H. Enatsu, A. Takenaka, and M. Okazaki, ibid. 43, 575 (1978).

[2] An exhaustive review of the localization problem up to the 1970s was given by A.J. Kálnay, in Problems in the Foundations of Physics, ed. M. Bunge (Springer-Verlag, Berlin, 1971).

[3] J.E. Johnson, Phys. Rev. 181, 1775 (1969).

[4] E.C.G. Stückelberg, Helv. Phys. Acta 14, 322 (1941); 14, 558 (1941); 15, 23 (1942). 
[5] J.P. Aparicio, F.H. Gaioli, and E.T. Garcia Alvarez, Interpretation of the evolution parameter of the Feynman parametrization of the Dirac equation (submitted to Phys. Lett. A); J.P. Aparicio, F.H. Gaioli, and E.T. Garcia Alvarez, Formulación de la Mecánica Cuántica Relativista parametrizada con un tiempo propio (Anales AFA 4, in press).

[6] J.R. Fanchi, Am. J. Phys. 49, 850 (1980); Found. Phys. 11, 493 (1981); B. Thaller, Lett. Nuovo Cimento 31, 439 (1981).

[7] W.C. Davidon, Phys. Rev. 97, 1131 (1955); 97, 1139 (1955).

[8] C. Teitelboim, Phys. Lett. B 96, 77 (1980).

[9] A.O. Barut, Found. Phys. 18, 95 (1988).

[10] C.R. Stephens, Ann. Phys. (N.Y.) 181, 120 (1988).

[11] P.A.M. Dirac, Proc. R. Soc. London, Ser. A 110, 405 (1926); 110, 661 (1926).

[12] P.A.M. Dirac, Proc. R. Soc. London, Ser. A 117, 610 (1928).

[13] V. Fock, Phys. Z. Sowjetunion 12, 404 (1937).

[14] G. Beck, Rev. Fac. de Ciencias de Coimbra 10, 66 (1942).

[15] Y. Nambu, Prog. Theor. Phys. 5, 82 (1950).

[16] R.P. Feynman, Phys. Rev. 80, 440 (1950) (in this work Feynman discussed only the Fock parametrization); 84, 108 (1951); S.S. Schweber, Rev. Mod. Phys. 58, 449 (1986).

[17] J. Schwinger, Phys. Rev. 82, 664 (1951).

[18] G. Szamosi, Nuovo Cimento 20, 1090 (1961); 29, 677 (1963).

[19] Z. Grossmann and A. Peres, Phys. Rev. 132, 2346 (1963).

[20] H.C. Corben, Phys. Rev. 121, 1833 (1961).

[21] D.M. Fradkin and R.H. Good, Rev. Mod. Phys. 33, 343 (1961).

[22] J.H. Cooke, Phys. Rev. 166, 1293 (1968).

[23] H.E. Moses, Ann. Phys. (NY) 52, 444 (1969).

[24] M. Bunge and A.J. Kálnay, Prog. Theor. Phys. 42, 1445 (1969).

[25] J.E. Johnson, Phys. Rev. D 3, 1735 (1971); J.E. Johnson and K.K. Chang, ibid. 10, 2421 (1974). Notice that in these works the authors do not restrict the states onto the positive mass subspace as in Ref. [3.

[26] J.J. Aghassi, P. Roman, and R.M. Santilli, Phys. Rev. D 1, 2753 (1970). Further developments can be found in J.J. Aghassi, P. Roman and R.M. Santilli, J. Math. Phys. 11, 2297 (1970); P. Roman, J.J. Aghassi, and P.L. Huddleston, ibid. 13, 1852 (1972); and P. Roman and J.P. Leveille, ibid. 15, 2053 (1974).

[27] L.P. Horwitz and C. Piron, Helv. Phys. Acta 46, 316 (1973); L.P. Horwitz and Y. Lavie, Phys. Rev. D 26, 819 (1982); R. Arshansky, L.P. Horwitz, and Y. Lavie, Found. Phys. 13, 1167 (1983); R. Arshansky and L.P. Horwitz, J. Math. Phys. 30, 66 (1989).

[28] The spin $1 / 2$ case in RD was considered by L.P. Horwitz, C. Piron, and F. Reuse, Helv. Phys. Acta 48, 546 (1975); C. Piron and F. Reuse, ibid. 51, 146 (1978); F. Reuse, ibid. 51, 157 (1978); see also L.P. Horwitz and R. Arshansky, J. Phys. A: Math. Gen. 15, L659 (1982).

[29] R.E. Collins and J.R. Fanchi, Nuovo Cimento A 48, 314 (1978); J.R. Fanchi, Phys. Rev. D 20, 3108 (1979); J.R. Fanchi and W.J. Wilson, ibid. 13, 571 (1983); J.R.
Fanchi, Phys. Rev. A 34, 1677 (1986).

[30] The equivalence between RD and FSF for arbitrary spin was demonstrated by J.R. Fanchi, J. Math. Phys. 22, 794 (1981).

[31] L. Hostler, J. Math. Phys. 21, 2461 (1980).

[32] M. Henneaux and C. Teitelboim, Ann. Phys. (NY) 143, 127 (1982).

[33] A.O. Barut and N. Zanghi, Phys. Rev. Lett. 52, 2009 (1984); A.O. Barut and W. Thacker, Phys. Rev. D 31, (1985).

[34] A. Kyprianidis, Phys. Rep. 115, 2 (1987), and references cited therein.

[35] P. Droz-Vincent, Phys. Lett. A 134, 147 (1988).

[36] J.P. Aparicio et al., Anales AFA 3, 46 (1991); 3, 51 (1991).

[37] S. Sonego, Phys. Rev. A 44, 5369 (1991).

[38] The proper time thought as a quantum operator has also been considered. See, e.g., A.J. Kálnay and E. Mac Cotrina, Prog. Theor. Phys. 42, 1422 (1969).

[39] Later on, the first-order parametrized Dirac equation was also considered by Schwinger; see J. Schwinger, Phys. Rev. D 12, 3105 (1975).

[40] A. Messiah, Mécanique Quantique (Dunod, Paris, 1964), Chap. 20.

[41] F.H. Gaioli, E.T. Garcia Alvarez, and A.J. Kálnay, The localization problem. A reinterpretation of the FeynmanBunge covariant position for the relativistic electron (unpublished); see also Ref. [24] and the second work of Ref. 33 .

[42] H.C. Bhabha and H.C. Corben, Proc. R. Soc. London, Ser. A 178, 273 (1941); H.C. Corben, Nuovo Cimento 20, 529 (1961); H.C. Corben and P. Stehle, Classical Mechanics (Wiley, New York, 1960), Chap. 16.

[43] F.H. Gaioli and E.T. Garcia Alvarez, Classical and quantum equations of motion of spinning particles (unpublished).

[44] See, e.g., L.G. Yaffe, Rev. Mod. Phys. 54, 407 (1982).

[45] The position $X^{\mu}$ has been considered, among others, by R.P. Feynman, Quantum Electrodynamics (Benjamin, New York, 1961), lecture 11.

[46] M. Bunge, Nuovo Cimento 1, 977 (1955).

[47] See, e.g., J.J. Giambiagi, Nuovo Cimento 16, 202 (1960); and Refs. [45], [18], and [24].

[48] J. Hilgevoord and S.S. Wouthuysen, Nucl. Phys. 40, 1 (1963); see also D.M. Fradkin and R.H. Good, Jr., Nuovo Cimento 22, 643 (1961).

[49] M. Kolsrud, Nuovo Cimento 39, 504 (1965).

[50] L. Michel and A.S. Wightman, Phys. Rev. 98, 1190 (1955); see also K. Rafanelli and R. Schiller, ibid. 135, B279 (1964).

[51] More precisely, Bargmann and Wigner used the PauliLubánski [cf. J.K. Lubánski, Physica 9, 310 (1942)] polarization $-\frac{1}{4} \epsilon^{\mu \nu \rho \lambda} \sigma_{\nu \rho} p_{\lambda}$ to characterize the representation of the Poincaré group. See V. Bargmann and E.P. Wigner, Proc. Natl. Acad. Sci. U.S.A. 34, 211 (1948).

[52] The "scalar product" 23 was also considered by H. Rumpf, Gen. Rel. Grav. 10, 509 (1979); and a similar definition can be found in Ref. 32 .

[53] An indefinite metric with similar formal characteristics has been worked out by Fesbach and Villars in the stan- 
dard RQM for the Klein-Gordon equation; see H. Fesbach and F. Villars, Rev. Mod. Phys. 30, 24 (1958).

[54] In another context, the indefinite metrics are well known in the literature; see, e.g., G. Barton, Introduction to Advanced Field Theory (Wiley, New York, 1963), Chap. 12.

[55] Some applications for strong interactions were treated by U. Heinz, Phys. Lett. 144B, 228 (1984). Our results can also be extrapolated to this case.

[56] J.P. Aparicio, F.H. Gaioli, and E.T. Garcia Alvarez, Proper time formulation of colored spinning particles (unpublished).

[57] L. Foldy, Phys. Rev. 87, 688 (1952); Rev. Mod. Phys. 30, 471 (1958); see also G. Salzman, Phys. Rev. 99, 973 (1955).

[58] J.P. Aparicio et al., Anales AFA 3, 83 (1991); see also W.A. Barker and Z.V. Chraplyvy, Phys. Rev. 89, 446 (1953).

[59] D. Bazeia, Lett. Nuovo Cimento 29, 228 (1980).

[60] G. Feinberg, Phys. Rev. 112, 1637 (1958); E.E. Salpeter, ibid. 112, 1643 (1958). 